\title{
Agenesis of the corpus callosum in two brothers
}

\author{
WILLIAM G WILSON, JAN M KENNAUGH, JANE P KUGLER, AND JAMES \\ F REYNOLDS
}

From the Department of Pediatrics, University of Virginia Medical Center, Charlottesville, Virginia 22908, USA.

SUMMARY Two brothers with developmental delay and unusual cranial configurations were found to have agenesis of the corpus callosum (ACC) by CT scan. Six published families in which ACC occurred in the absence of extracranial malformations are reviewed. No single mode of inheritance can account for all of these familial cases. This family illustrates the value of CT scanning in evaluating children with developmental delay.

The familial occurrence of agenesis of the corpus callosum (ACC), in the absence of extracranial malformations, is uncommon. We report two brothers with developmental delay and unusual head shape who have ACC. These cases demonstrate the usefulness of the CT scan in evaluation of children with developmental delay and cranial abnormalities.

\section{Case reports}

Case 1 was referred at the age of 19 months for evaluation of developmental delay and brachycephaly. He was the $2790 \mathrm{~g}$ product of a term pregnancy complicated by maternal vaginitis; there had been no exposure to medications. His nursery stay was prolonged because of fever which resolved without treatment. His growth and development for

Received for publication 8 December 1982. Accepted for publication 11 April 1983 the first 3 months were described as normal, but the 은 parents became concerned about progressive flatten- $\vec{\nabla}$ ing of the right posterior skull. His physician noted $\mathbb{D}$ developmental delay and cranial asymmetry at the $\frac{\Phi}{3}$ age of 5 months. Radiographs showed inward displacement of the right parietal and occipital bones without craniosynostosis. Developmental screening $\overrightarrow{0}$ at 10 months of age revealed marked delay in $\$$ areas, with most skills rated at the 3 to 4 month leve At the age of 19 months, his height $(78 \mathrm{~cm})$, weig $(9.5 \mathrm{~kg})$, and head circumference $(46 \mathrm{~cm})$ were less than the 5th centile for age. The cranium was brachycephalic with flattening of the right occiput. $\frac{\mathrm{D}}{\mathrm{D}}$ The cranial sutures were not ridged. Ophthalmolo- $\varrho$ gical examination, including fundoscopy, was normal. $\vec{\partial}$ The palmar creases, dermatoglyphs, and nails were unremarkable. Neurological examination showed generalised hypotonia and decreased deep tendon reflexes. Developmental testing using the Bayley
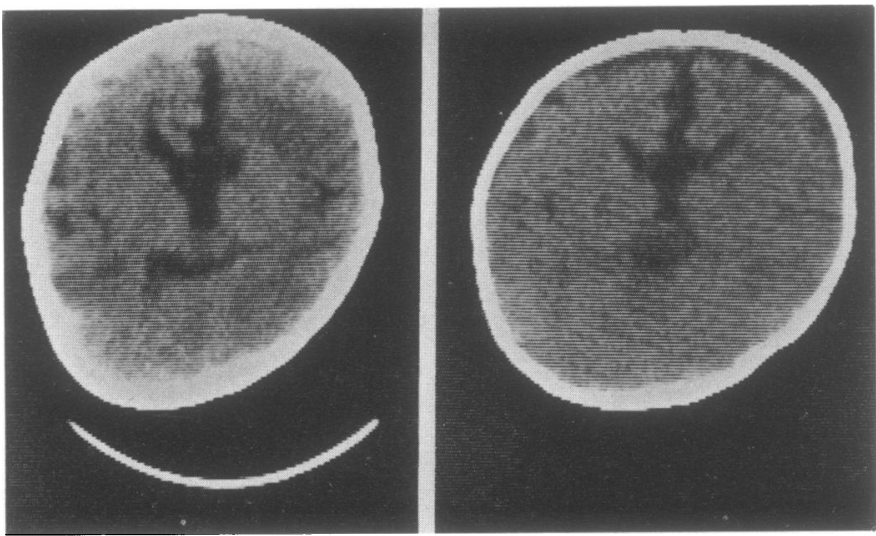

416
FIGURE Cranial CT scans of case 1 (left) and case 2 (right) showing absence of the corpus callosum, cortical atrophy, and cranial asymmetry. 
Scales of Infant Development placed him at the 4 month level. A cranial CT scan revealed generalised cortical atrophy and absence of the corpus callosum (figure).

Case 2, younger brother of case 1, was born following an uncomplicated term pregnancy. His birth weight and head circumference were $3520 \mathrm{~g}$ and $34.5 \mathrm{~cm}$, respectively. The mother was concerned because he had facial features resembling those of his brother. The neurological examination and cranial configuration were normal in the newborn period. Because of the problems of his older brother, case 2 received close medical follow-up. At 2 months of age, he exhibited delays in speech, cognition, and motor skills. At 5 months of age, his height was $62 \mathrm{~cm}$ (10th centile), weight $7.7 \mathrm{~kg}$ (60th centile), and head circumference $42.3 \mathrm{~cm}$ (30th centile). The right occiput was flattened with a ridging of the lambdoidal sutures. His face was broad with a wide flat nasal bridge and mildly downward slanting palpebral fissures. The optic discs were normal, but the left retinal vascular pattern was abnormal. His palmar creases and dermal ridge patterns were normal, but his fingernails were thickened and hyperconvex. Neurological examination revealed hypotonia and delayed gross motor, language, and speech skills. A CT scan of the head showed generalised cortical atrophy with prominent subarachnoid and interhemispheric spaces and agenesis of the corpus callosum (figure).

Studies performed on both patients included normal spine radiographs, serum electrolytes, and urinary amino-acids. A lymphocyte karyotype from patient 1 was normal.

There are no other family members with agenesis of the corpus callosum, although the mother claims to have two sibs with myelomeningoceles and associated hydrocephalus. The mother was aged 18 years and the father aged 24 years when case 1 was born. Both of them are in good health and are of normal intelligence and neurological function. There is no consanguinity.

\section{Discussion}

Although ACC can occur as part of a malformation syndrome, our patients did not have sufficient dysmorphic features, either separately or in common, to warrant the diagnosis of an existing syndrome or the proposal of a unique syndrome for them. There have been six reports of familial occurrence of ACC without extracranial malformations. ${ }^{1-6}$ These patients have presented with a variety of problems, including neonatal seizures, developmental delay, and adult macrocephaly without evidence of neurological impairment. Sibs were affected in five families: brothers in the family described by Zellweger, ${ }^{1}$ sisters in the families of Naiman and Fraser ${ }^{2}$ and Shapira and Cohen, ${ }^{4}$ and sibs of both sexes in the family reported by Cao et al..$^{5}$ The parents in these families were unrelated, with the exception of the family of Shapira and Cohen in which there was complex consanguinity. ${ }^{4}$ An affected father and son were described by Lynn et $a l^{6}{ }^{6}$ while Menkes $e t a l^{3}$ described a family in which only males were affected, including brothers, a half-brother, cousin, and uncle, all related through females. No single mode of inheritance can easily explain familial ACC in these families. It is possible that there is genetic heterogeneity in the causation of ACC, including both single gene and 'multifactorial' causes. The family which we report, with affected brothers, could represent any of these genetic aetiologies. Additional family studies, including studies of parents, may help clarify the genetic implications of ACC.

Attempts to define a neurological syndrome of ACC have shown a wide range of features associated with this malformation. Asymptomatic cases discovered at necropsy suggest that callosal absence is not necessarily associated with gross functional abnormalities. One explanation of the variety of neurological problems is that these findings are the result of related anomalies of the CNS and not of ACC alone. ${ }^{7}$

Before the availability of the CT scan, pneumoencephalography was the means of diagnosis of ACC in the living patient. This was not an innocuous procedure and was usually undertaken only when there was a high index of suspicion that an abnormality would be found. The non-invasive nature of the CT scan allows it to be used in patients with less striking findings. In a family described by Lynn et al, ${ }^{6}$ a child was evaluated because of macrocephaly and poor school performance and was found to have $\mathrm{ACC}$, as was his father who was macrocephalic but of normal intelligence. Such CT scan information will help to define the genetic implications of ACC. A CT scan should be considered as part of the diagnostic evaluation of any child with developmental delay or neurological abnormalities who has macrocephaly, unusual cranial configuration, or a family history of a subject with these problems.

\footnotetext{
References

1 Zellweger H. Agenesis corporis callosi. Helv Paediatr Acta 1952;7:136-55.

2 Naiman, J. Fraser FC. Agenesis of the corpus callosum. Arch Neurol 1955;75:182-5.

3 Menkes JH, Philippart M, Clark DB. Hereditary partial agenesis of the corpus callosum. Arch Neurol 1964;11: 198-208.

4 Shapira Y, Cohen T. Agenesis of the corpus callosum in two sisters.J Med Genet 1973;10:266-9.
} 
5 Cao A, Cianchetti C, Signorini E, Loi M, Sanna G, DeVirgilius S. Agenesis of the corpus callosum, infantile spasms, spastic quadriplegia, microcephaly and severe mental retardation in three siblings. Clin Genet 1977;12: $290-6$.

- Lynn RB, Buchanan DC, Fenichel GM, Freemon FR. Agenesis of the corpus callosum. Arch Neurol 1980;37: 444-5.

7 Warkany J, Lemire RJ, Cohen MM. Mental retardation and congenital malformations of the central nervous system. Chicago: Year Book Medical Publishers, 1980.

Correspondence and requests for reprints to Dr William G Wilson, Department of Pediatrics, University of Virginia Medical Center, Charlottesville, Virginia 22908, USA.

\section{Note added in proof}

Since the preparation of this manuscript a boy and his maternal uncle with ACC have been reported. (Kaplan P. X linked recessive inheritance of agenesis of the corpus callosum. J Med Genet 1983;20:122-4). 